(2) Open Access Full Text Article

REVIEW

\title{
Impact of extended-release dalfampridine on walking ability in patients with multiple sclerosis
}

This article was published in the following Dove Press journal:

Neuropsychiatric Disease and Treatment

28 April 201 I

Number of times this article has been viewed

\section{Keith C Hayes}

Department of Physical Medicine and Rehabilitation, The University of Western Ontario, London, ON, Canada
Correspondence: Keith C Hayes Parkwood Hospital/SJHC, 801

Commissioners Rd. E., London, ON, Canada N6C 5JI

$\mathrm{Tel}+\mathrm{I}$ 519-685-4292, ext. 42937

$\mathrm{Fax}+\mid$ 5|9-64|-725|

Email kchayes@uwo.ca
Abstract: Dalfampridine extended release (ER) $10 \mathrm{mg}$ is an oral tablet form of the potassium $\left(\mathrm{K}^{+}\right)$channel-blocking compounded dalfampridine, also known as fampridine, and chemically 4-aminopyridine or 4-AP, which received regulatory approval in the United States for the treatment of walking in patients with multiple sclerosis (MS) in January 2010. Two pivotal Phase 3 clinical trials demonstrated significant improvements in walking in patients with the four primary forms of MS following administration of dalfampridine ER tablets $10 \mathrm{mg}$ twice daily. The drug is thought to act by restoring conduction in focally demyelinated axons and by enhancing neurotransmission, thereby leading to improved neurological function. This review describes how dalfampridine represents a new pharmacotherapeutic approach to the clinical management of mobility impairment. It describes the mechanism of action and chemistry of dalfampridine ER, its pharmacokinetics, tolerability, and side effects, and the outcomes of multicenter trials showing its efficacy in improving walking speed. Clinician and patient global assessments, as well as patient self-assessment of the impact of MS on their gait disability, confirm clinically relevant benefit from the therapy. Patients tolerate the drug well and their improvement in terms of household and community ambulation, inferred from analysis of pooled data from several studies, is likely to translate into benefits in the performance of instrumental activities of daily living and a reduction in the neuropsychiatric burden of disease.

Keywords: dalfampridine ER, Ampyra ${ }^{\circledR}$, fampridine-SR, 4-aminopyridine, multiple sclerosis, demyelination

\section{Walking impairment in multiple sclerosis}

Impaired ability to walk constitutes one of the major disabling neuromuscular deficits associated with multiple sclerosis (MS) ${ }^{1-3}$ It is often the most visible sign of MS and has been reported to affect $80 \%$ of persons within 15 years of disease onset. ${ }^{1,4,5} \mathrm{At}$ this time there is a $40 \%$ probability of need for walking assistance and a $25 \%$ probability of need for a wheelchair. As a consequence of compromised activities of daily living that involve walking about the home or in the community, impairments of gait lead to loss of independence, decreased sense of self-worth due the external appearance of disablement, lost employment opportunities and reduced quality of life. ${ }^{3,6}$ The impact of mobility impairment on patient autonomy, caregiver burden, mood, and the costs of care are disproportionately high relative to those associated with other neurologic diseases $^{2}$ and compound the already heavy neuropsychiatric burden of the disease. ${ }^{2,7-9}$ As such, maintaining mobility is ranked as one of the highest priorities among patients with MS, regardless of disease duration or level of disability. ${ }^{3}$

Conventional approaches to the management of gait impairment primarily involve physical rehabilitation and the functional retraining of gait. This may be coupled with 
pharmacological management of symptoms that compromise gait, such as spasticity. Gait retraining is usually done by physical therapists and often involves the use of assistive mobility aids such as orthoses, canes, crutches, or walking frames. In advanced stages of the disease power scooters or wheelchairs may be prescribed..$^{10}$ A recent survey identified the fact that many patients elect not to use these devices; $37 \%$ of respondents did not use aids out of embarrassment and a further $36 \%$ reported that they did not use their prescribed aids as much as they should. ${ }^{11}$ Mobility aids do provide supports that constrain the disablement, but do not restore neuromuscular function per se. Similarly, pharmacologic agents for the management of spasticity reduce abnormalities of tone that impair locomotor capacity, but do so without reversing the underlying gait deficit. Antispastic and spasmolytic agents may also lead to unwanted central depressant actions of anergia and increased fatigue, thereby further contributing to the loss of mobility. Pharmacotherapeutics designed to slow progression of the disease, eg, interferons and glatiramer acetate, also provide little or no direct symptomatic relief of gait disorders, and recovery of function occurs only if the disease goes into remission.

Against the backdrop of this conventional management of impaired gait in MS, there has been considerable interest in the development of a new class of pharmacotherapeutics designed to reverse the underlying neurologic deficits associated with demyelination of axons within the CNS and thereby restore neurological function. The first drug in this class to be approved by the US Food and Drug Administration (FDA) is dalfampridine extended release (ER) (Acorda Therapeutics, Inc., New York), marketed under the name Ampyra $^{\circledR}$. In a series of Phase 2 and Phase 3 randomized clinical trials, dalfampridine ER $10 \mathrm{mg}$ tablets taken twice daily improved patients' speed on the Timed 25-Foot Walk test (T25FW). ${ }^{12-14}$ Based on the magnitude of the improvements in gait, and by reference to studies in other populations, the expected clinical benefits would be notable improvements in the patients' functional capacity to ambulate within their home or in the community. ${ }^{15}$ Several previous reviews of this drug have elaborated on the underlying pharmacology, the developmental history of the drug, and the outcome of clinical trials up to the time of regulatory approval by the FDA in January 2010. ${ }^{16-21}$

The US FDA determined that the previously adopted nonproprietary name "fampridine" was too similar to another approved medication and could lead to medical errors. The US Adopted Name Council therefore changed the nonproprietary name to "dalfampridine". In addition, the FDA determined that the term "extended release" will be used to refer to this formulation rather than the previously used "sustained release". These conventions have been employed in this manuscript. The drug is referred to by its International Nonproprietary Name of "fampridine" outside the US.

The present review briefly summarizes the pharmacological mechanism of action and pharmacokinetics of dalfampridine ER in individuals with MS. It also describes the quantitative and subjectively reported outcomes of clinical trials demonstrating efficacy for walking, safety, and tolerability. Unique to this review is commentary on the clinical significance of the observed therapeutic efficacy, and the current status of regulatory approval in other parts of the world. The review concludes with some perspective on how future research with dalfampridine might be directed to further explore the impact of the drug on neuropsychiatric burden in a more direct, rather than inferential, manner.

The information used to inform this review was largely derived from PubMed searches using the MeSH terms "4-aminopyridine", "fampridine", "dalfampridine", "ampyra", "human", "clinical trials", "multiple sclerosis", and identifying publications in English from 1985 to 2011. The search was completed March 15, 2011 and a total of 101 published articles were retrieved. Primary consideration was given to the original reports of Phase 2 and 3 clinical trials with supplemental information derived from subsequent reviews, meta-analyses, editorials, conference proceedings and abstracts. No attempt was made herein to independently conduct a formal systematic review of the quality of evidence emerging from these publications; however precis of both the critical analyses and the rebuttals are described in order to retain impartiality of this review. Information on mechanism of action and pharmacological properties was derived from studies involving animal models of demyelination. The FDA website (www.accessdata.fda.gov) provided access to prescribing information, tolerability, and adverse effects of dalfampridine ER. The Biogen Idec website (www.biogenidec.com) provided information on the regulatory status of the drug in Europe. Biogen Idec has the license to market dalfampridine ER outside the United States.

\section{MS, demyelination, and the mechanism of action of dalfampridine}

MS is primarily an inflammatory disorder of the central nervous system (CNS) in which focal lymphocytic infiltration leads to damage of myelin and axons. ${ }^{22}$ Demyelination of axons within the CNS is the most diagnostic neuropathology 
of MS in the early stages of the disease, and this has prompted characterization of MS as the archetypical demyelinating disease. Myelin deficits may show up as vacuolation, degeneration of the inner lamellae, focal thinning, disseminated foci of demyelination, or incomplete remyelination. These myelin deficits are thought to arise initially from $T$ cell, macrophage, and antibody-mediated oligodendrogliopathy and later from ischemic or toxic virus, or metabolic processes. ${ }^{23,24}$ As the disease progresses, there is commensurately more evidence of axonal pathology, with a distribution that varies with clinical phenotype. Axonal pathology accounts for the nonreversible nature of the neurological deficits and some of the neuropsychiatric symptoms in the progressive forms of disease. ${ }^{25-29}$

Myelin deficits lead to exposure of paranodal, fast, voltage-gated potassium $\left(\mathrm{K}^{+}\right)$channels, and the attendant abnormal $\mathrm{K}^{+}$conductance impairs action potential electrogenesis, repetitive axonal discharge, and propagation. ${ }^{30-32}$ Axonal conduction deficits that arise from demyelination underlie the initial clinical presentation of each of the four primary classifications of MS: relapsing-remitting, primary-progressive, secondary-progressive, and progressive-relapsing. Prominent among the neurologic deficits are visual disturbances, fatigue, and motor and sensory deficits including weakness, incoordination, and spasticity, all of which contribute to impaired balance and mobility, and compound independently impaired emotional and cognitive states.

Dalfampridine is a broad spectrum potassium $\left(\mathrm{K}^{+}\right)$channel blocking agent, with the capacity to improve conduction across demyelinated internodes in axons of the central nervous system. It has dose-dependent specificity for different types of $\mathrm{K}^{+}$channels. ${ }^{33}$ Dalfampridine prolongs the duration of the $\mathrm{Na}^{++}$action current at the internode; this increases the safety factor for conduction (ie, the ratio of action current generated by an impulse to the minimum amount of action current needed to maintain conduction) and thus can reverse conduction block due to focal demyelination. This mode of action has been established in various ex vivo and in vivo axonal preparations and animal models of demyelination. ${ }^{34-38}$ $\mathrm{K}^{+}$channel blocking compounds also enhance neuroneuronal and neuromuscular transmission by increasing presynaptic and end-terminal calcium $\left(\mathrm{Ca}^{++}\right)$influx. ${ }^{39,40}$ Collectively these properties are thought to underlie the restored axonal conduction and neurological function reported in patients with MS and other demyelinating disorders.

\section{Chemistry of dalfampridine}

Dalfampridine is also known by its chemical name, 4-aminopyridine, and is a member of a family of mono-amino and di-amino derivatives of pyridine, with a molecular structure of $\mathrm{C}_{5} \mathrm{H}_{6} \mathrm{~N}_{2}$. The pyridine ring has an amino substitution in the 4 position as shown in Figure 1. The molecular weight of dalfampridine is $94.12 .{ }^{41}$ An important feature of dalfampridine ER that distinguishes it from the compounded, immediate-release formulations of 4-aminopyridine is its prolonged pharmacokinetic half-life. The extended-release technology, developed by Élan Pharma International Ltd. (EPIL) and termed MXDAS ${ }^{\mathrm{TM}}$, consists of a proprietary polymer matrix that controls release by diffusion resulting in lower peak serum levels and longer duration of action. ${ }^{42}$

\section{Pharmacokinetics of dalfampridine ER in patients with MS}

The extended-release form of dalfampridine was developed commercially to overcome some of the pharmacokinetic and safety concerns associated with immediate-release formulations of the drug which had become available from compounding pharmacies. ${ }^{43,44}$ Concerns also existed about sustained-release forms of the drug available from compounding pharmacies, as they lacked quality control over manufacturing and distribution processes. Most notably, toxicity was associated with the high peak plasma concentrations of the immediate-release formulation $^{45}$ and the potency of the sustained-release forms was inconsistent, leading to risks associated with irregular dosing. Commercial development of the extended-release tablet form, with lower peak plasma concentrations, was designed to mitigate these risks and to ensure quality control and bring good manufacturing practices to the formulation and distribution of the drug. Reducing dosing requirements of dalfampridine ER to twice daily, instead of 3 to 5 times per day for immediate-release formulations, would also be expected to enhance patient compliance with oral medication.

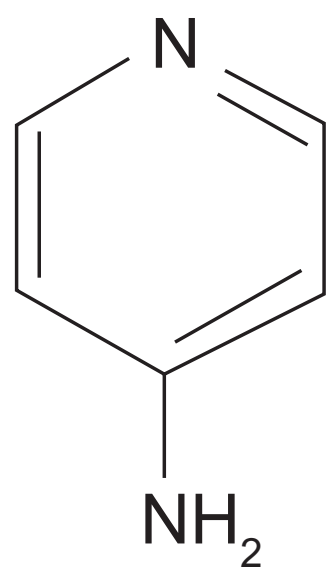

Figure I Molecular structure of 4-aminopyridine. 
The basic pharmacokinetics of an extended release formulation of dalfampridine in MS were initially reported by Bever et al. ${ }^{46}$ Twelve patients with Extended Disability Status Scale (EDSS) scores of 4 to 7.5 participated in an open-label study in which they received escalating doses $(7.5 \mathrm{mg}$ to $25 \mathrm{mg}$ every 12 hours) of dalfampridine. The mean time-to-peak and serum half-life were 5.0 and 5.2 hours, respectively, and maximum tolerable doses varied from $10 \mathrm{mg}$ twice daily to $25 \mathrm{mg}$ twice daily. These parameters were subsequently confirmed and elaborated by Vollmer and Henney ${ }^{47}$ in a multicenter, openlabel, evaluation of dose-proportionality in 24 patients with clinically definite MS. The pharmacokinetics of single escalating doses of dalfampridine ER (5-20 mg) are summarized in Table 1. In a second study, the steady state pharmacokinetic properties of dalfampridine ER $20 \mathrm{mg}$ twice daily were evaluated in the same patients over a 14-day period and compared with their single-dose study results. ${ }^{48}$ The pharmacokinetic parameters on day 1 replicated those of the single-dose study. The time to maximum plasma concentration $\left(\mathrm{T}_{\max }\right)$ values did not differ between days 1,8 , and 15 in the steady-state study, and the values found in the single-dose study (range 3.25-3.78 hours). The maximum plasma concentration $\left(\mathrm{C}_{\max }\right)$ values on day 8 and 15 were significantly higher than those on day 1 , and in the single-dose study reflected the accumulation of dalfampridine with multiple dosing. With the exception of one case of severe nausea, all adverse events in this study were mild to moderate and there were no significant changes in clinical laboratory values, vital signs, physical examination, or cardiac function (QTc intervals). This study provided support for the use of twice-daily dosing, approximately 12 hours apart, in this population. The absence of harmful effects on cardiac function has been confirmed elsewhere. ${ }^{49}$

In other studies, dalfampridine ER has been shown to be completely absorbed from the gastrointestinal gut following oral administration and to be almost completely (95.9\%) eliminated unchanged via the kidneys $(0.5 \%$ recovered from feces).$^{50}$ Small percentages are eliminated as the metabolite 3-hydroxy-4-aminopyridine (4.3\%) and 3-hydroxy-4-aminopyridine sulfate (2.6\%), neither of which is pharmacologically active at $\mathrm{K}^{+}$channels. ${ }^{50,51}$ Dalfampridine ER has a relative bioavailability of $96 \%$ compared with an aqueous oral solution. Plasma protein binding is low $(1 \%-3 \%)$ and the drug has an apparent volume of distribution of $2.6 \mathrm{~L} / \mathrm{kg}$. There are no indications of abnormal liver enzymes associated with the use of dalfampridine ER and the drug does not adversely interact with baclofen $(10 \mathrm{mg})$ or interferon- $\beta-1 b$ (subcutaneous injections of 8 million units), two medications commonly prescribed for use by MS patients. ${ }^{51}$ Dalfampridine ER kinetics were not affected by coadministration of these drugs. ${ }^{51}$

With respect to the interaction of dalfampridine ER with other drugs, it is noted in the prescribing information ${ }^{51}$ that in vitro data with human liver microsomes showed that dalfampridine ER was not a direct or time-dependent inhibitor of CYP1A2, CYP2A6, CYP2B6, CYP2C8, CYP2C9, CYP2C19, CYP2D6, or CYP3A4/5. Dalfampridine ER is not likely to affect the pharmacokinetics of drugs that are substrates of these enzymes. Other in vitro studies with cultured human hepatocytes with $0.025 \mu \mathrm{M}, 0.25 \mu \mathrm{M}, 2.5 \mu \mathrm{M}$, and $25 \mu \mathrm{M}$ dalfampridine ER had little or no effect on CYP1A2, CYP2B6, CYP2C9, CYP2C19, CYP2E1, or CYP3A4/5 enzyme activities. Consequently, the potential for dalfampridine ER to induce human hepatocytes at therapeutic concentrations is remote. In vitro, dalfampridine ER is not a substrate or an inhibitor for the p-glycoprotein transporter. The pharmacokinetics of dalfampridine ER are unlikely to be affected by drugs that inhibit the p-glycoprotein transporter, and dalfampridine ER is not likely to affect the pharmacokinetics of drugs that are substrates of the p-glycoprotein transporter.

In patients with renal impairment, accumulation of drug may occur with repeated administration. The drug should be used cautiously in individuals with mild renal impairment (creatinine clearance $[\mathrm{CrCl}] 51-80 \mathrm{~mL} / \mathrm{min}$ ) as the risk of seizures in these patients is unknown and the drug is not appropriate for patients with moderate or severely impaired renal function $(\mathrm{CrCl} \leq 50 \mathrm{~mL} / \mathrm{min})$. Plasma levels of

Table I Dalfampridine pharmacokinetic parameters

\begin{tabular}{|c|c|c|c|c|}
\hline \multicolumn{5}{|c|}{ Value by dalfampridine dose, mean (SE) } \\
\hline $\begin{array}{l}\text { Pharmacokinetic } \\
\text { parameter }\end{array}$ & $5 \mathrm{mg}$ & $10 \mathrm{mg}$ & $15 \mathrm{mg}$ & $20 \mathrm{mg}$ \\
\hline $\mathrm{T}_{\max }, \mathrm{h}$ & $3.88(0.20)$ & $3.92(0.25)$ & $3.36(0.25)$ & $3.57(0.25)$ \\
\hline $\mathrm{C}_{\max }, \mathrm{ng} / \mathrm{mL}$ & $13.13(0.64)$ & $25.23(1.39)$ & $37.00(1.99)$ & $49.23(3.16)$ \\
\hline$A \cup C, n g \cdot h / m L$ & I22.I (9.4) & $252.2(15.6)$ & $394.4(22.2)$ & $5 \mathrm{II.3(27.7)}$ \\
\hline $\mathrm{AUC}_{0 \rightarrow \infty}, \mathrm{ng} \cdot \mathrm{h} / \mathrm{mL}$ & $146.4(10.0)$ & $283.2(18.0)$ & $428.9(25.6)$ & $546.7(31.4)$ \\
\hline
\end{tabular}

Copyright $\odot$ 2009. Elsevier. Adapted with permission from Vollmer T, Henney HR. Pharmacokinetics and tolerability of single escalating doses of fampridine sustained-release tablets in patients with multiple sclerosis: a phase I-I I, open-label trial. Clin Ther. 2009;3I: 2206-22 I4.47 
dalfampridine ER in these patients may approach those seen at a dose of $15 \mathrm{mg}$ twice daily, a dose that may be associated with an increased risk of seizures. ${ }^{52}$ Because patients with renal impairment would require a dose lower than $10 \mathrm{mg}$ twice daily and no strength smaller than $10 \mathrm{mg}$ is available, dalfampridine ER is contraindicated in patients with moderate to severe renal impairment. Elderly patients are more likely to have decreased renal function and it is particularly important to know the estimated $\mathrm{CrCl}$ in these patients before initiating treatment with dalfampridine ER. Estimated $\mathrm{CrCl}$ should be known before initiating any treatment with dalfampridine ER.

The risks of dalfampridine ER and pregnancy are classified as Pregnancy Category C. ${ }^{51}$ There are no adequate and well-controlled studies of dalfampridine ER in pregnant women. Administration of dalfampridine ER to animals during pregnancy and lactation resulted in decreased offspring viability and growth at doses similar to the maximum recommended human dose of $20 \mathrm{mg}$ /day. Dalfampridine ER should be used during pregnancy only if the potential benefit justifies the potential risk to the fetus. ${ }^{51}$

\section{Tolerability and adverse events (side effects)}

The treatment-emergent adverse events associated with the use of dalfampridine ER are summarized in Table 2. The table reports pooled data from one Phase 2 and two Phase 3 studies and includes placebo data for comparison. ${ }^{51}$ Generally the drug is well tolerated by the majority of individuals within the recommended dosing of $10 \mathrm{mg}$ twice daily; most adverse events are mild and resolve without management within hours or a few days. Mild dizziness, gastrointestinal discomfort, and some agitation or wakefulness are frequently encountered. At higher doses, eg, at 20 to $30 \mathrm{mg}$ twice daily, the risk of more serious and intolerable adverse events increases. The most serious risk is for the induction of epileptiform seizures, eg, focal, tonic-clonic, and complex partial, and individuals who have a history or indicated risk of seizures should not expose themselves to further risk. In one of the Phase 2 trials, ${ }^{13} 2$ out of a total of 159 patients experienced seizures while taking $20 \mathrm{mg}$ twice daily; 1 of these patients had accidentally overdosed on 2 occasions, and experienced 2 partial complex seizures. There are no reports of recurrent seizures being induced by dalfampridine-ER. In the most recent Phase 3 clinical trial of dalfampridine ER $(n=239)$, 4 patients in the placebo group and 4 patients in the dalfampridine ER group withdrew because of adverse events. ${ }^{14}$ The largest proportional differences between treatment and placebo groups were found in adverse events of insomnia, headache, dizziness, nausea, back pain, and balance disorder, as reported using MedDRA terms (Medical Dictionary for Regulatory Activities). The majority of treatment-related events were mild or moderate in intensity, transient, and did not lead to discontinuation from the study.

Patient education about the dangers of using immediateor extended-release forms of dalfampridine, in a nonregulated or uncontrolled manner, or with accidental overdose, is clearly warranted as serious adverse events have been documented. ${ }^{43,45,53,54}$ The same applies to the risk of selfdosing dalfampridine ER above the prescribed limits particularly in the circumstance when some evidence of efficacy is present within the prescribed dose and individuals seek to gain greater benefit. Attention to the prescribing information for dalfampridine ER, medication labeling, and directions for use is essential. ${ }^{51}$ The multilevel Risk Evaluation and Mitigation Strategy (REMS) that Acorda Therapeutics, Inc. has implemented, involving medication guide and communication plan, is described on the FDA website. $^{55}$

\section{Randomized clinical trials showing efficacy in improving walking ability}

A series of uncontrolled Phase 1 and controlled Phase 2 clinical trials of dalfampridine and dalfampridine ER, conducted over a 20-year span, established some indications of efficacy for a variety of neurological impairments associated with MS and generally supported the safety and tolerability of the drug. ${ }^{12,56-63}$

In 2008, Goodman et al ${ }^{12}$ reported a Phase 2 parallel-group dose-comparison trial in which subjects were randomly assigned to receive dalfampridine-ER $10 \mathrm{mg}$ twice daily $(\mathrm{n}=52), 15 \mathrm{mg}(\mathrm{n}=50), 20 \mathrm{mg}(\mathrm{n}=57)$ or placebo $(\mathrm{n}=47)$. A 2 -week dose-escalation period was followed by a 12-week stable-dose phase and a 1-week dose-reduction phase. The percentage change in the $\mathrm{T} 25 \mathrm{FW}$ test was the primary outcome. The average increase in walking speed was $23.5 \%$ (10 mg), 26.0\% (15 mg), 15.8\% (20 mg), and $12.8 \%$ (placebo). The dalfampridine ER treatments did not yield significant improvement over placebo. A subsequent "responder" analysis revealed that the percentage of subjects exhibiting an a priori specified improvement of $>20 \%$ were higher in the dalfampridine ER-treated groups $(35.3 \%$ [10 mg], 36.0\% [15 mg], and 38.6\% [20 mg], respectively) compared with the $8.5 \%$ responding to placebo. As with the primary outcome these results did not attain statistical significance, and there was no indication of a dose-response, 
Table 2 Adverse reactions occurring in $\geq 2 \%$ of patients treated with dalfampridine ER tablets and more frequently than placebo-treated patients in clinical trials

\begin{tabular}{lll}
\hline & $\begin{array}{l}\text { Placebo } \\
(\mathbf{N}=\mathbf{2 3 8})\end{array}$ & $\begin{array}{l}\text { Ampyra } \\
\text { I O mg every I 2 h } \\
(\mathbf{N}=\mathbf{4 0 0})\end{array}$ \\
\hline Urinary tract infection & 8 & 12 \\
Insomnia & 4 & 9 \\
Dizziness & 4 & 7 \\
Headache & 4 & 7 \\
Nausea & 3 & 7 \\
Asthenia & 4 & 7 \\
Back pain & 2 & 5 \\
Balance disorder & I & 5 \\
Multiple sclerosis relapse & 3 & 4 \\
Paresthesia & 3 & 4 \\
Nasopharyngitis & 2 & 4 \\
Constipation & 2 & 3 \\
Dyspepsia & I & 2 \\
Pharyngolaryngeal pain & 1 & 2 \\
\hline Notes: & &
\end{tabular}

Notes: Numbers represent percentage of subjects experiencing the adverse reaction. Adapted from United States Food and Drug Administration: Prescribing Information for Ampyra $^{T M}$ (dalfampridine) extended release tablets. www.accessdata.fda.gov/ drugsatfda_docs/label/.../022250s000 lbl.pdf.Accessed March 24, 201 I. ${ }^{51}$

but they did engender some optimism that the drug may be helpful to a subset of individuals with mobility impairment. The only endpoint attaining significance was the change from baseline in lower extremity strength, which showed an improvement of roughly $15 \%$ over placebo in both the 10 - and 15-mg dose groups.

The responder type analyses prompted $\mathrm{Kryscio}^{64}$ to observe that the investigators had discovered an "innovative method for identifying consistent responders to the drug, providing clinicians with a promising tool for treating a subset of MS patients". Kryscio $^{64}$ also questioned whether this was a true positive finding or an example of data massaging. While acknowledging that responder analyses are acceptable within the context of regulatory approval by the FDA, Kryscio ${ }^{64}$ also noted that the failure to detect statistically significant outcomes may have been due to a lack of statistical power. A major disadvantage of the responder analysis approach is reduced power relative to that based on group averages ${ }^{65}$ In this particular study, to make the responder analysis the primary endpoint and meet FDA requirements on comparing multiple doses with placebo, the investigators would have needed to accrue 195 patients per group to show a doubling of responders with equivalent power ( $80 \%$ power with an adjusted type I error rate of 0.016 ).

Two pivotal Phase 3 trials were then designed to rigorously evaluate the efficacy of dalfampridine ER in enhancing performance on the $\mathrm{T} 25 \mathrm{FW}$ in patients with clinically definite MS. The responder analysis was selected as the analytic method to be employed. In order to directly assess the clinical relevance of the outcomes, the 12-Item MS Walking Scale (MSWS-12) was also administered. ${ }^{66}$ This scale has been shown to be a reliable and valid patient-based measure of the impact of MS on walking and has been shown to be more responsive than other walking-based scales. ${ }^{66}$ These trials were conducted under the auspices of FDA Special Protocol Assessments.

The first Phase 3 trial (MS-F203) $)^{13}$ was a double-blind, placebo-controlled, parallel-group study conducted in 33 centers in the United States and Canada. Participants received dalfampridine ER $10 \mathrm{mg}$ twice daily or placebo, over a 14-week treatment period, with each arm of the study preceded by a 2-week placebo run-in. All participants were followed for 4 weeks after completion of the double-blind phase of the trial. A total of 301 patients was randomized into the study, on a 3:1 ratio, to receive treatment drug or placebo, and of these 283 completed all study visits. The primary outcome measure, the T25FW, was evaluated 4 times prior to treatment, 4 times during treatment, and twice in the follow-up period. Patients were allowed to use an assistive device while walking, as long as the same device was used consistently across visits. The a priori defined responder analysis was then employed to determine the number of patients who showed a consistent improvement in walking speed during the double-blind treatment period. The primary efficacy endpoint was calculated using a modified intentionto-treat analysis. Secondary outcomes of efficacy related to neuromuscular benefits included measures of lower extremity muscle strength (Lower Extremity Manual Muscle Test or LEMMT) and spasticity (Ashworth Score). The Subject Global Impression (SGI), Clinician Global Impression (CGI), and MSWS-12 were also used as secondary outcomes to validate the clinical meaningfulness of the response criterion of consistent improvement on the T25FW.

The primary result of MS-F203 was that the proportion of timed walk responders in the dalfampridine ER-treated group (34.8\%) was significantly greater than in the group given placebo (8.3\%). Responders to treatment had an average increase in walking speed of $25.2 \%$ during the course of treatment whereas the placebo group showed a $4.7 \%$ improvement. The increase in walking speed was maintained throughout the double-blind treatment period. The responders reported greater improvement in the self-assessment of walking capacity (MSWS-12) than nonresponders. A significant increase in leg strength was observed in dalfampridine ER responders compared with subjects in the placebo group. The responder group also showed greater improvement in MSWS-12 scores than nonresponders. These results 
supported the a priori FDA-approved efficacy criterion that treatment with dalfampridine ER $10 \mathrm{mg}$ twice daily would significantly increase the chance of having a positive response of improved walking speed that is consistent over time and perceived subjectively as a meaningful improvement in ambulatory deficits.

The second Phase 3 trial (MS-F204) was also a doubleblind, placebo-controlled, parallel group study in patients with clinically definite MS. This was conducted across 39 centers in the United States and Canada and involved 239 patients who were randomized to treatment with dalfampridine ER $10 \mathrm{mg}$ twice daily or placebo on a $1: 1$ ratio. For inclusion, participants had to have a T25FW time between 8 and 45 seconds, and the T25FW was again used as the primary outcome measure. The treatment duration was shortened to 9 weeks because of the previously demonstrated stability of beneficial changes in walking speed over a 3-month period.

The results of this trial established that the proportion of T25FW responders was higher in the dalfampridine ER group (42.9\%) compared with the placebo group (9.3\%) and the average improvement in walking speed for responders was $24.7 \%$ or $0.51 \mathrm{ft} / \mathrm{s}$. The increase in walking speed among dalfampridine ER-treated responders was maintained across the double-blind treatment period and was reversed with treatment discontinuation. The average changes from baseline in MSWS-12 score during the double-blind treatment period were -6.04 for responders compared with 0.85 for nonresponders, indicating a significant reduction in selfassessed ambulatory disability among responders. SGI, and CGI scores both confirmed significant benefit for responders. This study thus provided class 1 evidence that dalfampridine ER produced clinically meaningful improvement in walking ability in a subset of people with MS.

Subsequent to completion of these two Phase 3 studies, pooled analyses have been undertaken combining the results of one of the Phase 2 trials, with comparable design, and the two Phase 3 trials. The conclusions drawn were that the efficacy of dalfampridine ER, as defined by the timed-walk responder rate, was independent of demographics, disease characteristics, and use of immunomodulatory drugs. ${ }^{67}$ Moreover, the benefits of dalfampridine ER were seen across a wide range of baseline deficits in walking ability. ${ }^{15}$ Using a scale derived from the post-stroke population, which characterized functional walking into household ambulation $(<1.3 \mathrm{ft} / \mathrm{s})$, limited community ambulation $(1.3-2.6 \mathrm{ft} / \mathrm{sec})$, or full community ambulation $(>2.6 \mathrm{ft} / \mathrm{s})$, Edwards et al ${ }^{15}$ reported that a substantial proportion of responders in the three studies sufficiently improved their walking speed to represent a change from less to more function within a class and in many cases individuals would be expected to move from one ambulation class to another.

\section{The role of dalfampridine therapy for gait disorders in MS}

The primary indication for dalfampridine ER is for walking in MS patients regardless of the severity of deficit and disease characteristics. Pharmacotherapy would be done in conjunction with the use of mobility aids and would be complementary to symptomatic management strategies such as the use of antispastic agents. The use of dalfampridine ER concurrently with baclofen has been shown to be safe in the course of the clinical studies, which did not exclude concurrent treatment; indeed the reported antispastic properties of dalfampridine ER reported in some of the Phase 2 and 3 trials, and evidenced by improved scores on the Ashworth scale, may indicate the possibility of reducing the dosing requirements of anti-spastic medications that are being used, though this has not been established by clinical studies. The demonstrated efficacy and safety of dalfampridine ER in trials in which concurrent use of immunomodulatory, disease-modifying agents were allowed suggests that parallel pharmacotherapeutic strategies may be employed.

The reported clinical benefits of dalfampridine ER extend beyond those for which class 1 evidence exists. There is persuasive experimental evidence of improvements in leg strength and reductions in spasticity, both of which may enable greater independence with respect to transfers and activities of daily living. Indeed, Kachuck ${ }^{18}$ has reported on a number of benefits revealed on clinical examination and by clinical experience but not necessarily amenable to, or captured in, formal clinical trial protocols. These include improvements in endurance or fatigability, visual acuity, bladder/bowel/sexual dysfunction. Improvements in mental sharpness, problem solving and reading comprehension have also been reported anecdotally. Some experimental support for these findings has been noted. ${ }^{56-62}$ Clearly, clinical judgement will prevail as to how such benefits to these symptoms, if observed, influence concurrent management approaches.

\section{Challenges to the purported clinical benefit of dalfampridine}

There have been two recently documented challenges to the acceptance of dalfampridine ER as a therapeutic agent with meaningful clinical benefit. In the first, an editorial 
published in Annals of Neurology in 2010, by Hauser and Johnston, ${ }^{68}$ the authors maintained that the drug "improved the 25 foot walk time by an average of less than one second; 0.88 seconds to be exact in the first trial and 0.5 seconds in the second. Although statistically significant, the benefit appears modest for a treatment that costs $\$ 13,000$ annually". The authors also noted the continued risk of seizures and implied there was little gain from this medication over the immediate-release formulations available through compounding pharmacies at one-fiftieth of the cost. A rebuttal by Cohen and Blight, ${ }^{69}$ on behalf of Acorda Therapeutics, and published in January 2011, focused on some inaccuracies in the editorial concerning the development of the drug, highlighted the risks of serious dosing errors associated with compounded formulations of 4-AP, ${ }^{43,44}$ and addressed what they considered to be an inaccurate reflection of the average change in walking times experienced by the patients. They noted that the results reported in the editorial "appear to have been derived by an unusually complex, post hoc manipulation of a subset of the trial data". The a priori specified analysis of the study data focused on reproducible improvements in walking speed, acknowledging the day-to-variability in functional capacity experienced by individuals with MS. They noted that in the $37.6 \%$ of responders to dalfampridine ER there was an average $25 \%$ improvement in walking speed compared to $6.5 \%$ in placebo-treated patients and $7.0 \%$ in dalfampridine ER-treated nonresponders. The clinical meaningfulness of this improvement in the responder group was reflected by a significant reduction in self-assessed walking disability, evident in the MSWS-12.

In the second challenge, which occurred about the same time as the rebuttal by Cohen and Blight, the Committee for Medicinal Products for Human Use (CHMP), the European agency responsible for conducting the initial assessment of medicines for which an EU-wide marketing authorization is sought, rejected the application for dalfampridine ER (submitted by Biogen Idec under the name Fampyra ${ }^{\mathrm{TM}}$ ). ${ }^{70} \mathrm{CHMP}$ was also not convinced that Fampyra's effect on the walking speed was a meaningful benefit for patients. Moreover, they argued that the effect on walking speed could not be linked to meaningful improvements such as better coordination, balance, or stamina or increased range of motion. The Committee was not persuaded that the medicine's benefits outweighed its side effects. The Committee also noted the lack of adequate long-term data on the medicine's benefits and safety as well as data on some groups of patients, such as the elderly and patients with epilepsy or heart problems.
Biogen Idec is appealing this decision at the time of this review going to press.

\section{Impact of dalfampridine ER on the neuropsychiatric burden of diseases in MS}

The neuropsychiatric burden of MS is multifaceted and complex..$^{7-9}$ It includes neuropathology-related impairments of emotional control and cognitive processes brought about by demyelination and/or axonal pathology in various locations within the brain sub-serving these functions. Benedict et al, ${ }^{28}$ for example, have reported that measures of tissue atrophy including whole-brain and central atrophy are especially well correlated with and predictive of cognitive impairment. The burden also involves disability-related, "reactive" processes such as mood disorders associated with diagnosis, experienced disability and its social consequences, and concern for the future because of the progressive nature of the disease and the interaction with the normal functional decline of aging; ie, "accelerated degradation". ${ }^{2,71}$ Compounding the burden on the patient is the huge impact of their needs on caregivers, ${ }^{72}$ both contracted caregivers and loved ones, and the economics of managing their disability, often when their employment has been compromised. ${ }^{2}$

How does dalfampridine ER impact these states? There is no empirical evidence from controlled trials to directly address these issues. Inference drawn from the Phase 3 trials and earlier studies does suggest that improved mobility may mitigate the dependence on others for some activities of daily living, including personal care and homemaking tasks, and may enable greater community involvement or extended employment. Such benefits would be expected to translate to fewer feelings of dependence and burden of the stigma associated with disability. The extent to which this becomes clinically and personally meaningful will likely depend on the specific disease state and circumstances of each patient.

The neurobiological mechanism of action of dalfampridine ER in restoring conduction in demyelinated axons is unlikely to be confined to the sensory and motor pathways involved in gait, even if there exist subtle differences in $\mathrm{K}^{+}$ channel typology and distribution in different parts of the CNS. ${ }^{33}$ Indeed one could reasonably project that disseminated foci of demyelination throughout the CNS all represent viable targets for the drug action. In this case, one might expect to find that dalfampridine ER is capable of reversing demyelination-based cognitive and emotional deficits and 
thereby attenuating the neuropsychiatric burden directly. Smits et al, ${ }^{73}$ in a small $(\mathrm{n}=20)$ randomized, double-blind, crossover trial, examined the effects of 2 weeks treatment with immediate-release oral 4-aminopyridine (dalfampridine) on cognitive function in patients with definite MS. Although there was a trend for improved performance with the study medication for two of the neuropsychological tests $(10 / 36$ delayed recall and the Paced Auditory Serial Addition Test of attention), the study failed to demonstrate significant group effects of dalfampridine on cognitive function. The extent to which medication-induced improvements in performance will be feasible and able to yield clinical benefit, however, must be weighed in the context of the evolving awareness of distinct patterns and regional distribution of gray matter damage that are associated with cognitive impairment in MS patients. ${ }^{27}$ The correspondence between lesion formation and gray matter atrophy distribution varies in the different forms of MS. Thus the degree to which dalfampridine may be useful will depend on the degree to which demyelination, as distinct from axonal pathology, contributes to the cognitive dysfunction and this may vary among individuals and among clinical phenotypes.

\section{Implications for further research}

Two obvious lines of enquiry emerge from the above discussion of the impact of dalfampridine ER on walking ability and the corollary of attenuating the neuropsychiatric burden. The first is to more directly examine the impact of drug-induced improvements in walking on instrumental activities of daily living, employment, or other rehabilitation outcomes. Although challenging because of the multivariate nature of factors influencing performance and outcome, it is through empirically demonstrating benefit in these areas that some of the reactive burden may be lifted. Accompanying those forms of benefit would likely be demonstrable enhancements in the quality of life. ${ }^{74}$ The second line of enquiry would be to directly evaluate the efficacy of the drug in influencing biomarkers or other indices of cognitive or neuropsychiatric impairment. These types of studies may provide focus for further Phase 2 or 3 studies, or postmarketing Phase 4 investigations that will enable new indications to be identified.

As a first-generation pharmaceutical designed to reverse demyelination-induced conduction deficits, dalfampridine ER is likely to spawn further developments with novel $\mathrm{K}^{+}$channel blocking agents with similar or more refined mechanisms of action. New insights are emerging about the large family of voltage-gated $\mathrm{K}^{+}$ion channels. ${ }^{75}$ Elaboration of the properties of these channels and their relationship to disease and neurological dysfunction is helping to define new therapeutic targets. $^{76}$

\section{Summary}

Dalfampridine ER is the first of a new class of pharmacotherapeutics designed to restore neurological function compromised by central demyelination. It has received approval from the FDA in the United States for the improvement of walking in people with MS and is currently pending regulatory approval in Canada. The FDA has required additional studies investigating the efficacy of lower doses of dalfampridine ER, notably $5 \mathrm{mg}$ tablets. In Europe the drug (under the name Fampyra) has been denied approval for marketing by the CHMP because of what was considered small benefit relative to risk, a decision currently under appeal by Biogen Idec. The improvements in gait brought about by the drug are evident as increased walking speed on a timed walking test (T25FW), positive global assessments by clinician and patient, and reduced walking disability as reflected in the patient self-assessed MSWS-12. Since mobility impairments contribute greatly to the patient burden of disease with respect to activities of daily living, employment, selfimage, and mood, improvements in gait would be projected to mitigate some of the reactive neuropsychiatric burden of disease. Whether or not there are direct effects of the drug in reversing demyelination-induced axonal conduction deficits underlying emotional and cognitive impairment remains to be determined by further research.

\section{Disclosure}

$\mathrm{K}$ C Hayes $\mathrm{PhD}$ received and exercised stock options for consulting with Acorda Therapeutics, Inc., New York, the company that sponsored the clinical trials of Fampridine-SR (dalfampridine extended release) and is now marketing the product in the USA under the name Ampyra ${ }^{\mathrm{TM}}$.

\section{References}

1. Baum HM, Rothschild BB. Multiple sclerosis and mobility restriction. Arch Phys Med Rehabil. 1983;64:591-596.

2. Dunn J. Impact of mobility impairment on the burden of caregiving in individuals with multiple sclerosis. Expert Rev Pharmacoeconomics Outcome Res. 2010;10(4):DOI:10.1586/ERP.10.34.

3. Sutliff MH. Contribution of impaired mobility to patient burden in multiple sclerosis. Curr Med Res Opin. 2010;26:109-119.

4. Myhr KM, Riise T, Vedeler C, et al. Disability and prognosis in multiple sclerosis: demographic and clinical variables important for the ability to walk and awarding of disability pension. Mult Scler. 2001; 7:59-65.

5. Whetten-Goldstein K, Sloan FA, Goldstein LB, et al. A comprehensive assessment of the cost of multiple sclerosis in the United states. Mult Scler. 1998;4:419-425. 
6. Salter AR, Cutter GR, Tyry T, et al. Impact of loss of mobility on instrumental activities of daily living and socioeconomic status in patients with MS. Curr Med Res Opin. 2010;26:493-500.

7. Pinkston JB, Alekseeva N. Neuropsychiatric manifestations of multiple sclerosis. Neurol Res. 2006;28:284-290.

8. Ghaffar O, Feinstein A. The neuropsychiatry of multiple sclerosis: a review of recent developments. Curr Opin Psychiatry. 2007;20: 278-285.

9. Paparrigopoulos T, Ferentinos P, Kouzoupis A, et al. The neuropsychiatry of multiple sclerosis: focus on disorders of mood, affect and behaviour. Int Rev Psychiatry. 2010;22:14-21.

10. Souza A, Kelleher A, Cooper R, et al. Multiple sclerosis and mobilityrelated technology: sytematic review of literature. J Rehab Res Dev. 2010;47:201-212.

11. Harris Interactive. Key findings from two new multiple sclerosis surveys [internet]. New York (NY): National MS Society and Acorda Therapeutics; 2008. http:/www.nationalmssociety.org/news/newsdetail/ download.aspx?id=1018.

12. Goodman AD, Brown TR, Cohen JA, et al; Fampridine MS-F202 Study Group. Dose comparison trial of sustained-release fampridine in multiple sclerosis. Neurology. 2008;71:1134-1141.

13. Goodman AD, Brown TR, Cohen JA, et al. Sustained-release oral fampridine in multiple sclerosis: a randomised, double-blind, controlled trial. Lancet. 2009;373:732-738.

14. Goodman AD, Brown TR, Edwards KR, et al. A phase 3 trial of extended release oral dalfampridine in multiple sclerosis. Ann Neurol. 2010;68:494-502.

15. Edwards K, Brown T, Schapiro AD, et al. Dalfampridine extended release tablets improve walking speed across a wide range of baseline deficits: pooled data from three placebo-controlled studies in patients with multiple sclerosis. [abstract] 62nd Annual Meeting of the American Academy of Neurology; 2010 Apr 10-17; Toronto.

16. Hayes KC. Fampridine-SR for multiple sclerosis and spinal cord injury. Exp Rev Neurother. 2007;7:453-461.

17. Korenke AR, Rivey MP, Allington DR. Sustained-release fampridine for symptomatic treatment of multiple sclerosis. Ann Pharmacol. 2008;42: 1485-1465.

18. Kachuck NJ. Sustained release oral fampridine in the treatment of multiple sclerosis. Expert Opin Pharmacother. 2009;10:2025-2035.

19. Bever CT, Judge SI. Sustained-release fampridine for multiple sclerosis. Expert Opin Investig Drugs. 2009;18:1013-1024.

20. Chwieduk CM, Keating GM. Dalfampridine extended release in multiple sclerosis. CNS Drugs. 2010;24:883-891.

21. Goodman AD, Hyland M. Dalfampridine in multiple sclerosis. Drugs Today (Barc). 2010;46:635-639.

22. Compston A, Coles A. Multiple sclerosis. Lancet. 2008;372: 1502-1517.

23. Barten LJ, Allington DR, Procacci KA, Rivey MP. New approaches to the management of multiple sclerosis. Drug Des Dev Ther. 2010;24: 343-366.

24. Kleinschnitz C, MEuth SG, Kiesseir BC, Wiendl H. Immunotherapeutic approaches in MS: update on pathophysiology and emerging agents 2006. Endocr Metab Immune Disord Drug Targets. 2007;7:35-63.

25. Dutta R, Trapp BD. Mechanisms of neuronal dysfunction and degeneration in multiple sclerosis. Prog Neurobiol. 2010 Oct 12. [Epub ahead of print].

26. Siffrin V, Vogt J, Radbruch H, Nitsch R, Zipp F. Multiple sclerosis candidate mechanisms underlying CNS atrophy. Trends Neuroscience. 2010;33:202-210.

27. Riccitelli G, Rocca MA, Pagani E, et al. Cognitive impairment in multiple sclerosis is associated to different patterns of gray matter atrophy according to clinical phenotype. Hum Brain Mapp. 2010. [Epub ahead of print].

28. Benedict RH, Carone DA, Bakshi R. Correlating brain atrophy with cognitive dysfunction, mood disturbances, and personality disorder in multiple sclerosis. J Neuroimaging. 2004;14(3 Suppl):36S-45S.
29. Rocca MA, Riccitelli G, Rodegher M, et al. Functional MR imaging correlates of neuropsychological impairment in primary-progressive multiple sclerosis. Am J Neuroradiol. 2010;31:1240-1246.

30. McDonald WI, Sears TA. The effects of experimental demyelination on conduction in the central nervous system. Brain. 1970;93:583-598.

31. Waxman SG. Conduction in myelinated, unmyelinated, and demyelinated fibers. Arch Neurol. 1977;34:585-589.

32. Waxman SG. Membranes, myelin, and the pathophysiology of multiple sclerosis. N Engl J Med. 1982;306:1529-1533.

33. Judge SI, Bever CT Jr. Potassium channel blockers in multiple sclerosis: Neuronal Kv channels and effects of symptomatic treatment. Pharmacol Ther. 2006;111:224-259.

34. Bostock H, Sherratt RM, Sears TA. Overcoming conduction failure in de-myelinated nerve fibres by prolonging action potentials. Nature. 1978;274:385-387.

35. Sherratt RM, Bostock H, Sears TA. Effects of 4-aminopyiridine on normal and demyelinated mammalian nerve fibres. Nature. 1980;283: $570-572$.

36. Bostock H, Sears TA, Sherratt RM. The effects of 4-aminopyridine and tetraethylammonium ions on normal and demyelinated mammalian nerve fibres. J Physiol. 1981;313:301-315.

37. Bowe CM, Kocsis JD, Targ EF, Waxman SG. Physiological effects of 4-aminopyridine on demyelinated mammalian motor and sensory fibers. Ann Neurol. 1987;22:264-268.

38. Targ EF, Kocsis JD. 4-aminopyridine leads to restoration of conduction in demyelinated rat sciatic nerve. Brain Res. 1985;328:358-361.

39. Jankowska E, Lundberg A, Rudomin P, Sykova E. Effects of 4-aminopyridine on synaptic transmission in the cat spinal cord. Brain Res. 1982;240:117-129.

40. Lundh H. Effects of 4-aminopyridine on neuromuscular transmission. Brain Res. 1978;153:307-318.

41. Uges DRA, Huizinga T. 4-aminopyridine; analysis of the substance and a method for the preparation of a solution for injection in man. Pharm Acta Helv. 1981;56:158-162.

42. United States Food and Drug Administration: Medication Guide for Ampyra $^{\mathrm{TM}}$ (dalfampridine) Extended Release Tablets. http://www.fda. gov/downloads/Drugs/DrugSafety/UCM199168.pdf. Accessed March 24, 2011.

43. Burton JM, Bell CM, O'Connor PW. 4-aminopyridine toxicity with unintentional overdose in four patients with multiple sclerosis. Neurology. 2008;71:1833-1834.

44. Schwam E. Severe accidental overdose of 4-aminopyridine due to compounding pharmacy error. J Emerg Med. 2009:DOI:10.1016. 2009.04.037.

45. Bever CT Jr, Young D, Anderson PA, et al. The effects of 4-aminopyridine in multiple sclerosis patients: results of a randomized, placebo-controlled, double-blind, concentration-controlled, crossover trial. Neurology. 1994;44:1054-1059.

46. Bever CT Jr, Young D, Tierney D, et al. The pharmacokinetics and tolerability of a slow-release formulation of 4-aminopyridine in multiple sclerosis patients. Neurology. 1995;45(Suppl 4):A351.

47. Vollmer T, Henney HR. Pharmacokinetics and tolerability of single escalating doses of fampridine sustained-release tablets in patients with multiple sclerosis: a phase 1-11, open-label trial. Clin Ther. 2009;31: 2206-2214.

48. Vollmer T, Blight AR, Henney HR. Steady-state pharmacokinetics and tolerability of orally administered fampridine sustained-release 10-mg tablets in patients with multiple sclerosis; a 2-week, open-label, follow-up study. Clin Ther. 2009;31:2215-2223.

49. March B, Cardi T. Assessment of the cardiac safety of fampridine-SR sustained-release tablets through a thorough QT/QTc evaluation at therapeutic and subtherapeutic doses in healthy individuals. Expert Opin Inv Drugs. 2009;18:1807-1815.

50. Blight AR, Henney HR. Pharmacokinetics of ${ }^{14} \mathrm{C}$-radio-activity after oral intake of a single dose of ${ }^{14} \mathrm{C}$-labelled fampridine (4-aminopyridine) in healthy volunteers. Clin Ther. 2009;31:328-335. 
51. United States Food and Drug Administration: Prescribing Information for Ampyra ${ }^{\mathrm{TM}}$ (dalfampridine) extended release tablets. www.accessdata.fda.gov/drugsatfda_docs/label/.../022250s000 lbl.pdf. Accessed March 24, 2011.

52. Smith W, Swan S, Marbury T, Henney H 3rd. Single-dose pharmacokinetics of sustained-release fampridine (Fampridine-SR) in healthy volunteers and adults with renal impairment. J Clin Pharmacol. 2010;50:151-159.

53. Pickett TA, Enns R. Atypical presentation of 4-aminopyridine overdose. Ann Emerg Med. 1996;27:382-385.

54. Stork CM, Hoffman RS. Characterization of 4-aminopyridine in overdose. J Toxicol Clin Toxicol. 1994;32:583-587.

55. United States Food and Drug Administration: Risk Evaluation and Mitigation Strategy (REMS) for Ampyra (Dalfampridine) $10 \mathrm{mg}$ tablets. http://www.accessdata.fda.gov/drugsatfda_docs/ label/2010/022250s000REMS.pdf. Accessed March 24, 2011.

56. Davis FA, Stefoski D, Rush J. Orally administered 4-aminopyridine improves clinical signs in multiple sclerosis. Ann Neurol. 1990;27: 186-192.

57. Stefoski D, Davis FA, Faut M, Schauf CL. 4-Aminopyridine improves clinical signs in multiple sclerosis. Ann Neurol. 1987;21:71-77.

58. Stefoski D, Davis FA, Fitzsimmons WE, Luskin SS, Rush J, Parkhurst GW. 4-Aminopyridine in multiple sclerosis: Prolonged administration. Neurology. 1991;41:1344-1348.

59. Van Diemen HA, Polman CH, van Dongen TM, et al. The effect of 4-aminopyridine on clinical signs in multiple sclerosis: a randomized, placebo-controlled, double-blind, cross-over study. Ann Neurol. 1992;32:123-130.

60. Van Diemen HA, Polman CH, van Dongen MM, et al. 4-Aminopyridine induces functional improvement in multiple sclerosis patients: a neurophysiological study. J Neurol Sci. 1993;116:220-226.

61. Polman CH, Bertelsmann FW, van Loenen AC, Koetsier JC. 4-aminopyridine in the treatment of patients with multiple sclerosis. Long-term efficacy and safety. Arch Neurol. 1994;51:292-296.

62. Schwid SR, Petrie MD, McDermott MP, Tierney DS, Mason DH, Goodman AD. Quantitative assessment of sustained release 4-aminopyridine for symptomatic treatment of multiple sclerosis. Neurology. 1997;48:817-821.
63. Goodman AD, Cohen JA, Cross A, et al. Fampridine-SR in multiple sclerosis: a randomized, double-blind, placebo controlled, dose ranging study. Mult Scler. 2007;13:357-368.

64. Kryscio RJ. Fampridine for MS responders: Clinically relevant or hypothesis generating? Neurology. 2008;71:1130-1131.

65. Altman DG, Royston P. The cost of dichotomizing continuous variables. BMJ. 2006;332:1080.

66. Hobart JC, Riazi A, Lampring DL, Fitzpatrick R, Thompson AJ. Measuring the impact of MS on walking ability: The 12-Item MS Walking Scale (MSWS-12). Neurology. 2003;60:31-36.

67. Brown T, Schapiro R, Edwards K, et al. Response to treatment with dalfampridine extended release tablets in patients with multiple sclerosis is independent of baseline patient characteristics and concomitant immunomodular therapy [abstract] 62nd Annual Meeting of the American Academy of Neurology; 2010 Apr 10-17; Toronto.

68. Hauser SL, Johnston SC. 4-Aminopyridine: new life for an old drug. Ann Neurol. 2010;68:A8-A9.

69. Cohen R, Blight AR. 4-Aminopyridine: new life for an old drug. Reply. Ann Neurol. 2011;69:218-220.

70. Biogen Idec website. www.biogenidec.com. Accessed March 24, 2011.

71. Hayes KC, Wolfe DL, Trujillo SA, Burkell JA. On the interaction of disability and aging: Accelerated degradation models and their influence of projections of future care needs and costs for personal injury litigation. Disabil Rehabil. 2010;32:424-428.

72. Figved N, Myhr KM, Larsen JP, Aarsland D. Caregiver burden in multiple sclerosis: the impact of neuropsychiatric symptoms. J Neurol Neurosurg Psychiatry. 2007;78:1097-1102.

73. Smits RC, Emmen HH, Bertelsmann FW, Kulig BM, van Loenen AC, Polman $\mathrm{CH}$. The effects of 4-aminopyridine on cognitive function in patients with multiple sclerosis: a pilot study. Neurology. 1994;44: 1701-1705.

74. Zwibel HL. Contribution of impaired mobility and general symptoms to the burden of multiple sclerosis. Adv Ther. 2009;26:1043-1057.

75. Judge SI, Smith PJ, Stewart PE, Bever CT Jr. Potassium channel blockers and openers as CNS neurologic therapeutic agents. Recent Pat CNS Drug Discov. 2007;2:200-228.

76. Wulff H, Castle NA, Pardo LA. Voltage-gated potassium channels as therapeutic targets. Nat Rev Drug Discov. 2009;8:982-1001.
Neuropsychiatric Disease and Treatment

\section{Publish your work in this journal}

Neuropsychiatric Disease and Treatment is an international, peerreviewed journal of clinical therapeutics and pharmacology focusing on concise rapid reporting of clinical or pre-clinical studies on a range of neuropsychiatric and neurological disorders. This journal is indexed on PubMed Central, the 'PsycINFO' database and CAS, and is the official

\section{Dovepress}

journal of The International Neuropsychiatric Association (INA). The manuscript management system is completely online and includes a very quick and fair peer-review system, which is all easy to use. Visit http://www.dovepress.com/testimonials.php to read real quotes from published authors. 